\title{
In-vitro efficacy of polymyxin B with rifampin, colistin and doxycycline against extensively drug resistant Acinetobacter baumannii
}

\author{
Abdul Hannan ${ }^{1}$, Fizza Khalid ${ }^{2 \star}$ and Muhammad Usman Arshad ${ }^{3}$ \\ Department of Microbiology, University of Health Sciences, Lahore, Pakistan.
}

Accepted 27 December, 2013

\begin{abstract}
Acinetobacter baumannii is an opportunistic Gram negative coccobacillus that can grow easily in moist as well as dry conditions. During the last decade, $A$. baumannii emerged as one of the most resistant opportunistic pathogens responsible for nosocomial infections including ventilator associated pneumonia. The bug remains an important and difficult to treat pathogen whose pan-drug resistant nature has created a serious challenge. This has restricted the choice of treatment modalities. Currently, it appears as if all the available antibiotics are failing against this pathogen while single antibiotic therapy is certainly not working anymore. Thus, there is a strong need, thus, to explore new regimens to combat this resistant organism. A wide range of various combinations of drugs should therefore be tested for their synergistic activity against this pathogen. This study was aimed to find some effective combinations against extensively drug resistant (XDR) $A$. baumannii by combining various antibacterials. The microdilution checkerboard titration method was used for this purpose and fractional inhibitory concentrations (FICs) were calculated. In-vitro synergy was found in polymyxin Bcolistin $(n=3 ; 15 \%)$ and polymyxin B-rifampin $(n=3 ; 15 \%)$ combinations. Only additive effect was noted with polymyxin B-doxycycline $(n=12 ; 60 \%)$, polymyxin B-rifampin $(n=11 ; 55 \%)$, and polymyxin $B-$ colistin ( $n=13 ; 65 \%)$. However, antagonism was detected in the polymyxin B-rifampin combination in one of the $\mathbf{2 0}$ strains evaluated for the purpose. Polymyxin B in combination with rifampin and colistin may be exploited against XDR $A$. baumannii. Synergy between polymyxin B and colistin have been demonstrated in only $15 \%$ of strains, this fully warrants the testing of more combinations.
\end{abstract}

Key words: Acinetobacter baumannii, extensively drug resistant, fractional inhibitory concentration.

\section{INTRODUCTION}

The genus Acinetobacter is a ubiquitous group of microorganisms (Giamarellou et al., 2008) and is found in the environment. Acinetobacter baumannii was considered to be a pathogen of low grade pathogenecity and was ignored whenever isolated from clinical specimens until the 1970s (Zarrilli et al., 2009). It has recently emerged as one of the most troublesome nosocomial pathogens globally and has become a major cause of health careassociated and community-acquired infections (Talbot et al., 2006; Davis et al., 2005). Management of MDR/XDR

*Corresponding author. E-mail: khalidfiza@rocketmail.com. Tel: 03334079326.

Abbreviations: A. baumannii, Acinetobacter baumannii; API 20NE, analytical profile index 20 Non-Enterobactericeae; ATCC, American type culture collection; FIC, fractional inhibitory concentration; FICI, fractional inhibitory concentration index; MBC, minimal bactericidal concentration; MDR, multi-drug resistant; MIC, minimal inhibitory concentration; PDR, pan-drug resistant; XDR, extensively drug resistant. 
A. baumannii infections is a big challenge for physicians and clinical microbiologist. The organism's capability to survive in hospital settings and to persist for long periods of time on various surfaces makes it a frequent cause of healthcare associated infections. Another problem regarding $A$. baumannii is its ability to cause a wide spectrum of infections which include wound infections, bacteremia, pneumonia, urinary tract infections, etc (Manchanda et al., 2010). A. baumannii is proven to have the capability to form biofilms that is believed to play central part in the process of colonization (Pour et al., 2011).

During the early 1970 s, the clinical isolates of $A$. baumannii were usually susceptible to various antibiotic classes (Bergogne-Berezin and Towner, 1996). However, since 1975, increasing resistance to almost all groups of antibacterials started appearing (Manchanda et al., 2010; Montefour et al., 2008). From the year 2000 to date, various combinations of antibiotics have been evaluated for their synergistic activity or otherwise to combat this resistant pathogen (Rodriguez-Hernandez et al., 2000; Montero et al., 2004; Saballs et al., 2006; Tan et al., 2011).

The focus of this study was to determine the synergistic effect of polymyxin B with either of the antibiotics: rifampin, colistin and doxycycline by the checkerboard microtitration technique.

\section{MATERIALS AND METHODS}

\section{Bacterial strains}

The extensively drug resistant $A$. baumannii (XDR-AB) phenotype was identified as an $A$. baumannii strain, resistant to all classes of the traditional antibiotics except tigecycline and polymyxin $B$ using the disc diffusion method according to Clinical and Laboratory Standards Institute (CLSI, 2012) recommendations. These strains were isolated from various clinical samples, and collected from various patients hospitalized in Lahore, Pakistan in 2012. The strains were identified by their morphological and biochemical characteristics and, later by using API 20-NE (BioMerieux, France). The selected strains were stored in microbanks at $-80^{\circ} \mathrm{C}$.

\section{Antimicrobial agents and minimal inhibitory concentration} determination

The base materials of antimicrobial agents used in combinations were: polymyxin B (Glaxosmith Kline pharmaceuticals), rifampin (Pacific pharmaceuticals), colistin (Forest pharmaceuticals) and doxycycline (Pfizer Global pharmaceuticals). Stock solutions of antibiotics were prepared in their respective solvents (water for polymyxin B, colistin and doxycycline, methanol for rifampin) according to the CLSI 2012 guidelines and stored at $-20^{\circ} \mathrm{C}$ for one week. Minimal inhibitory concentrations (MICs) of all strains for each antibiotic were determined by a standard agar dilution method. Bacterial inoculum equivalent to $0.5 \mathrm{McF}$ arland $\left(5 \times 10^{8}\right)$ was prepared and diluted 1:10 to achieve the final concentration of $5 \times 10^{7} \mathrm{CFU} / \mathrm{ml}$. The concentration range of various antibiotics was prepared; 0.125 to $8.0 \mu \mathrm{g} / \mathrm{ml}$ (polymyxin B and colistin), 0.5 to 64 $\mu \mathrm{g} / \mathrm{ml}$ (doxycycline), 0.06 to $64 \mu \mathrm{g} / \mathrm{ml}$ (rifampin) in Mueller Hinton agar. The plates were inoculated with the bacterial suspensions using multipoint inoculators (MAST Diagnostics UK). The same was incubated for $24 \mathrm{~h}$ at $37^{\circ} \mathrm{C}$. Lowest concentration at which bacterial growth was inhibited was noted after incubation (CLSI 2012). Escherichia coli ATCC 25922 was used as a control strain. MIC results were read and interpreted according to the CLSI breakpoint criteria for $A$. baumannii. Since there are no CLSI interpretation criteria of rifampin, available, relevant to $A$. baumannii, the breakpoints for this antibiotic were based on the MIC standards of CLSI for Gram positive bacteria (CLSI, 2012).

\section{Synergy testing}

The synergistic activity of the antibiotic combinations was determined using the microdilution checkerboard titration method. The range of concentrations was chosen according to the previously determined MIC of each antibiotic for each isolate. Concentrations used ranged from $0.06 \times$ MIC to $8 \times$ MIC for each antibiotic. The interpretation of the checkerboard synergy testing results was determined by the method of Orhan et al. (2005). FICs and $\mathrm{FICl}$ were calculated for each antimicrobial combination using the formulas below:

$\Sigma \mathrm{FIC}$ or $\mathrm{FICl}=\mathrm{FIC} \mathrm{A}+\mathrm{FIC} \mathrm{B}$

Where,

FIC $A=\frac{\text { MIC of drug } A \text { in the combination }}{\text { MIC of drug } A \text { alone }}$

and

FIC B $=\frac{\text { MIC of drug B in the combination }}{\text { MIC of drug B alone }}$

The combination was considered synergistic when the $\Sigma F I C$ was $\leq 0.5$, additive when the $\Sigma F I C$ was $>0.5$ to $\leq 1.0$ indifferent when the $\Sigma F I C$ was $>1.0$ to $<2$, and antagonistic when the $\Sigma F I C$ was $\geq 2$ (Orhan et al., 2005).

\section{RESULTS}

The detail of the various clinical materials from which $A$. baumannii was originally isolated is shown in Figure 1. The highest number of $A$. baumannii strains were isolated from central venous catheter tips $(n=9 ; 45 \%)$ followed by pus $(n=8 ; 40 \%)$, urine $(n=1 ; 5 \%)$, high vaginal swab $(n=1$; $5 \%$ ) and body fluids $(n=1 ; 5 \%)$. Major isolation from CVC tips is mainly due to the capability of $A$. baumannii to survive on dry as well as moist conditions and also grow well on tubings of catheters and ventilators. The second highest source was pus which indicates its ability to colonize open wounds and from where it can invade into the blood stream to cause life threatening bacteremia. All isolates were resistant to all the classes of antimicrobials except polymyxin B (100\% susceptible) and doxycycline ( $85 \%$ susceptible). However, all were resistant to colistin irrespective of their susceptibility or otherwise to polymyxin B and doxycycline. Their susceptibility patterns are shown in the Figure 2.

$\mathrm{MIC}_{90}$ for polymyxin $\mathrm{B}$, colistin, doxycycline and rifampin was $1,8,64$ and $2 \mu \mathrm{g} / \mathrm{ml}$ respectively. Their MIC ranges and susceptibility rates are shown in Table 1. MIC for ATCC E. coli 25922 for polymyxin B, colistin, doxycy- 


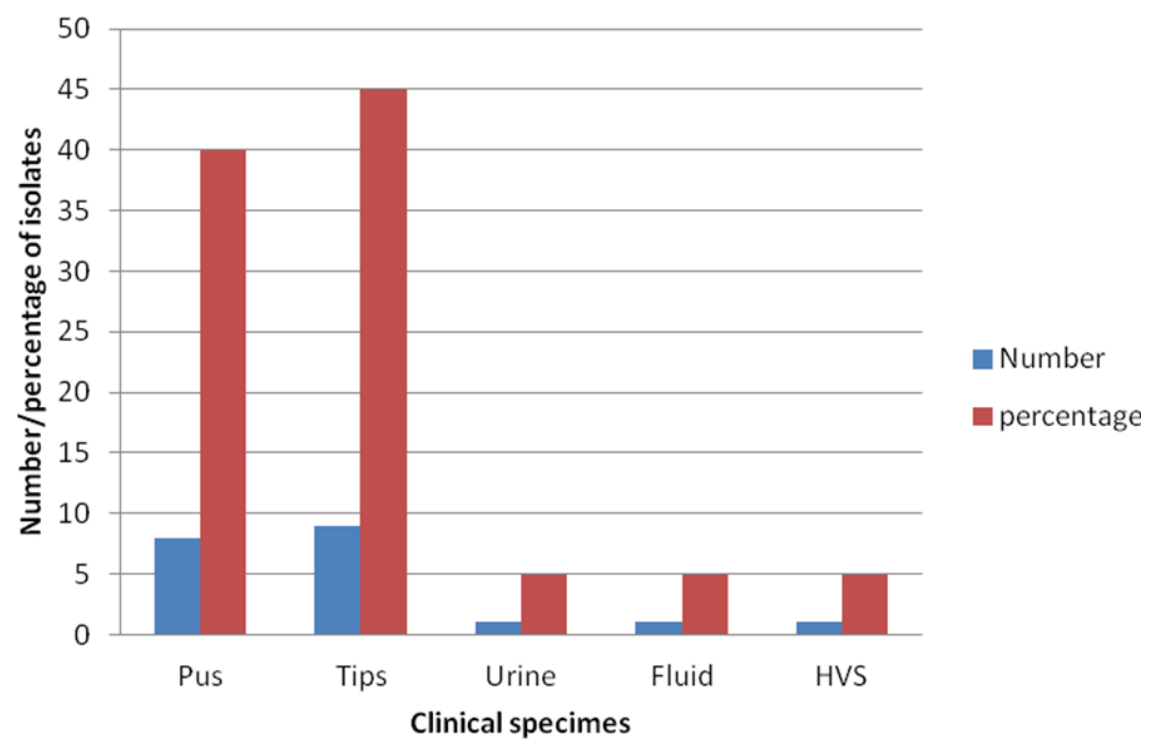

Figure 1. Sources of XDR A. baumannii isolates.

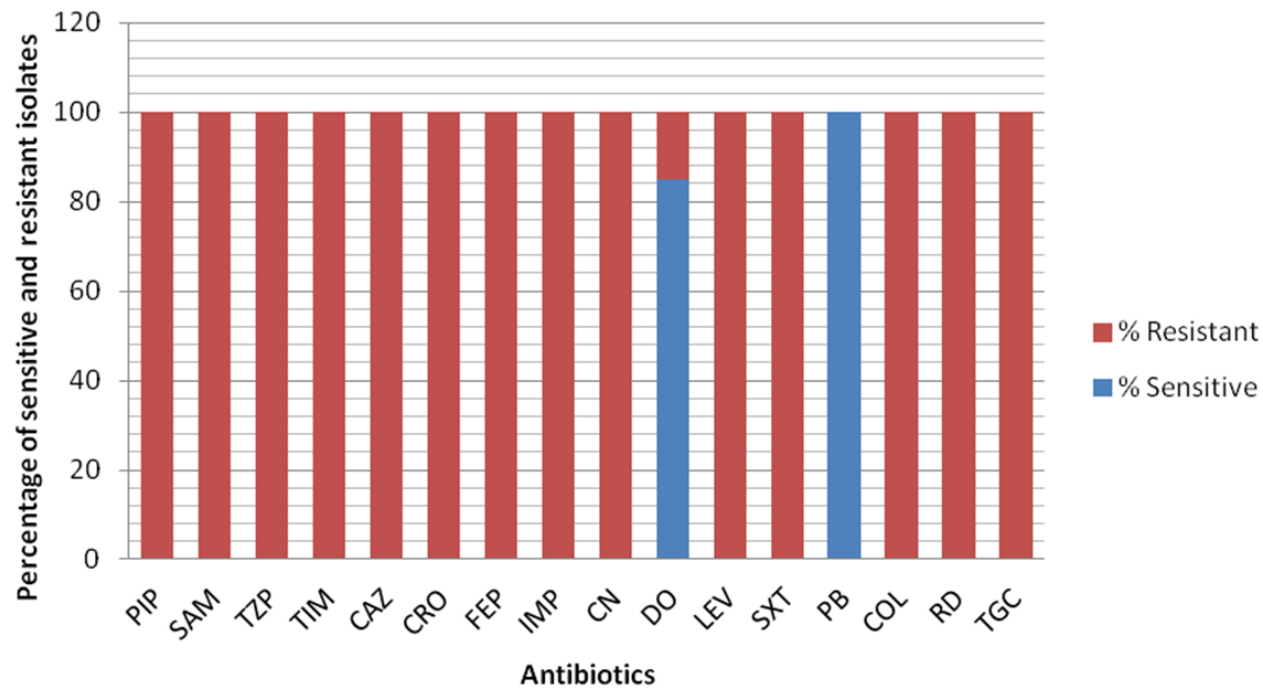

Figure 2. Susceptibility of $A$. baumannii isolates to various antibiotics. PIP: piperacillin, SAM: ampicillin-sulbactam, TZP: piperacillin-tazobactam, TIM: ticarcillin-clavulanic acid, CAZ: ceftazidime, CRO: ceftriaxone, FEP: cefepime, IMP: imipenem, CN: gentamicin, DO: doxycycline, LEV: levofloxacin, SXT: cotrimoxazole, PB: polymyxin B, COL: colistin, RD: rifampin, TGC: tigecycline.

cline, and rifampin are also shown in Table 1. MICs of XDR $A$. baumannii for four tested antibiotics by broth microdilution method are shown in Table 2.

The results of the microtitration checkerboard method are shown in Table 3. Three isolates (15\%): AB-02, AB14 , and $A B-20$ strains showed synergistic effect in polymyxin B-rifampin combination while $55 \%$ additive effect was seen in this combination. In the case of AB-02 and $A B-20$, the MIC of polymyxin $B$ in combination was reduced to one fourth as compared to the individual MICs against these isolates. The MIC of rifampin was reduced to one eighth and one fourth for $A B-02$ and $A B-20$ respectively. For the isolate $A B-14$, the MIC of polymyxin $B$ and rifampin was reduced to one eighth and one fourth respectively.

Indifference was detected in $25 \%$ of the isolates while AB-08 showed antagonism (5\%) in this combination of antimicrobials. The MIC of polymyxin B was increased 
Table 1. Minimal inhibitory concentrations (MICs), susceptibility rates and quality control (QC) ranges of XDR A. baumannii ( $\mathrm{n}=20)$ and $E$. coli ATCC 25922 versus 4 different antibiotics.

\begin{tabular}{|c|c|c|c|c|c|c|c|c|}
\hline \multirow{3}{*}{ Antibiotic } & \multicolumn{6}{|c|}{ XDR A. baumannii $(\mathrm{n}=20)$} & \multicolumn{2}{|c|}{ E. coli ATCC ${ }^{\text {a }} 25922(\mathrm{n}=1)$} \\
\hline & \multicolumn{3}{|c|}{ MIC $(\mu \mathrm{g} / \mathrm{ml})$} & \multicolumn{3}{|c|}{ Susceptibility rate $(\%)^{b}$} & \multirow{2}{*}{$\begin{array}{c}\text { MIC } \\
(\mu \mathrm{g} / \mathrm{ml})\end{array}$} & \multirow{2}{*}{$\begin{array}{c}\text { MIC QC Ranges }^{\mathrm{d}} \\
(\mu \mathrm{g} / \mathrm{ml})\end{array}$} \\
\hline & Range $^{c}$ & $\mathrm{MIC}_{50}$ & MIC $_{90}$ & Susceptible & Intermediate & Resistant & & \\
\hline Polymyxin B & 0.5 to 1.0 & 0.5 & 1.0 & 100 & - & - & 0.5 & $0.25-2.0$ \\
\hline Rifampin & 1.0 to 8.0 & 2.0 & 2.0 & - & - & - & 8.0 & $4.0-16$ \\
\hline Colistin & 4.0 to 8.0 & 4.0 & 8.0 & - & - & 100 & 1.0 & $0.25-2.0$ \\
\hline Doxycycline & 1.0 to 64 & 1.0 & 64 & 85 & - & 15 & 1.0 & $0.5-2.0$ \\
\hline
\end{tabular}

a: American Type Culture Collection; b: susceptibility was interpreted according to Clinical Laboratory Standard (CLSI) 2012 guidelines. C: Susceptibility range given by CLSI 2012 guidelines. d: Quality control ranges provided by CLSI 2012 guidelines against ATCC reference strain.

Table 2. Minimal inhibitory concentrations (MICs) of XDR A. baumannii $(\mathrm{n}=20)$ versus 4 different antibiotics by broth microdilution method.

\begin{tabular}{lcccc}
\hline \multirow{2}{*}{ Isolate no. } & \multicolumn{4}{c}{ MIC $(\boldsymbol{\mu g} / \mathbf{m l})$} \\
\cline { 2 - 5 } & Polymyxin B & Colistin & Doxycycline & Rifampin \\
\hline AB-01 & 0.5 & 1.0 & 0.5 & 1.0 \\
AB-02 & 0.5 & 2.0 & 32 & 2.0 \\
AB-03 & 1.0 & 2.0 & 0.5 & 1.0 \\
AB-04 & 0.5 & 1.0 & 0.25 & 1.0 \\
AB-05 & 0.5 & 1.0 & 0.25 & 1.0 \\
AB-06 & 0.5 & 2.0 & 0.5 & 1.0 \\
AB-07 & 1.0 & 1.0 & 0.5 & 1.0 \\
AB-08 & 0.5 & 2.0 & 0.25 & 1.0 \\
AB-09 & 0.5 & 1.0 & 0.5 & 2.0 \\
AB-10 & 0.5 & 1.0 & 0.5 & 1.0 \\
AB-11 & 0.5 & 2.0 & 0.25 & 2.0 \\
AB-12 & 0.5 & 4.0 & 0.5 & 1.0 \\
AB-13 & 0.5 & 2.0 & 0.5 & 1.0 \\
AB-14 & 0.5 & 2.0 & 32 & 8.0 \\
AB-15 & 0.5 & 2.0 & 0.5 & 2.0 \\
AB-16 & 1.0 & 2.0 & 0.25 & 2.0 \\
AB-17 & 0.5 & 2.0 & 0.25 & 1.0 \\
AB-18 & 0.5 & 2.0 & 0.25 & 1.0 \\
AB-19 & 0.25 & 2.0 & 0.5 & 1.0 \\
AB-20 & 1.0 & 2.0 & 64 & 8.0 \\
\hline
\end{tabular}

four times for $\mathrm{AB}-08$ in combination with rifampin and showed antagonism. In polymyxin B-colistin combination, three strains: (15\%) AB-06, AB-19, and AB-20 showed synergistic effect. In all the three isolates showing synergism, MIC of colistin was reduced by one fourth in combination with polymyxin B. For AB-19, MIC was dropped to one eighth, while for the rest of the two isolates it was reduced to one fourth in combination.

Additive effect in this combination was found to be $65 \%$, and indifference was $20 \%$ while there was no antagonism detected in this combination. In the case of polymyxin B-doxycycline combination, no synergism or antagonism was shown. Additive effect was $60 \%$ while the remaining $40 \%$ was indifference shown in this combination.

\section{DISCUSSION}

A. baumannii has become a major challenge due to its multiple drug resistance. In recent years, more and more cases of mortality and morbidity due to MDR/XDR $A$. baumannii have come to light (Queenan et al., 2012; Karaiskos et al., 2013).

Several studies have been done, both in-vitro and invivo, to demonstrate the synergism of two or more antibiotics in combination against resistant pathogens (Gunderson et al., 2003; Lim et al., 2011; Fiori and Van 
Table 3. Synergy test results of antibiotic combination by checkerboard microtitration method.

\begin{tabular}{|c|c|c|c|c|c|c|c|c|c|c|c|c|}
\hline \multirow[b]{2}{*}{$\begin{array}{l}\text { Strain } \\
\text { no. }\end{array}$} & \multicolumn{4}{|c|}{ PB - RD } & \multicolumn{4}{|c|}{ PB - COL } & \multicolumn{4}{|c|}{ PB - DO } \\
\hline & $\begin{array}{l}\text { Conc. } \\
\text { of PB }\end{array}$ & $\begin{array}{l}\text { Conc. } \\
\text { of RD }\end{array}$ & $\Sigma$ FIC & Activity & $\begin{array}{l}\text { Conc. } \\
\text { of PB }\end{array}$ & $\begin{array}{l}\text { Conc. } \\
\text { of COL }\end{array}$ & $\sum$ FIC & Activity & $\begin{array}{l}\text { Conc. } \\
\text { of PB }\end{array}$ & $\begin{array}{l}\text { Conc. } \\
\text { of DO }\end{array}$ & $\Sigma$ FIC & Activity \\
\hline AB-01 & MIC & $1 / 2 \mathrm{MIC}$ & 1.5 & ID & $1 / 2 \mathrm{MIC}$ & $1 / 2 \mathrm{MIC}$ & 1.0 & ADD & $1 / 2 \mathrm{MIC}$ & MIC & 1.5 & ID \\
\hline AB-02 & 1/4 MIC & 1/8 MIC & 0.375 & $S$ & 1/4 MIC & 1/2 MIC & 0.75 & ADD & 1/2 MIC & 1/4 MIC & 0.75 & ADD \\
\hline AB-03 & 1/2 MIC & 1/4 MIC & 0.75 & ADD & 1/2 MIC & MIC & 1.5 & ID & 1/2 MIC & 1/4 MIC & 0.75 & ADD \\
\hline AB-04 & MIC & 1/2 MIC & 1.5 & ID & 1/2 MIC & 1/4 MIC & 0.75 & ADD & 1/2 MIC & MIC & 1.5 & ID \\
\hline AB-05 & 1/2 MIC & 1/8 MIC & 0.625 & ADD & 1/2 MIC & 1/2 MIC & 1.0 & ADD & 1/2 MIC & MIC & 1.5 & ID \\
\hline AB-06 & 1/2 MIC & 1/4 MIC & 0.75 & ADD & 1/4 MIC & 1/4 MIC & 0.49 & $S$ & 1/2 MIC & 1/2 MIC & 1.0 & ADD \\
\hline AB-07 & 1/2 MIC & 1/4 MIC & 0.75 & ADD & 1/2 MIC & MIC & 1.5 & ID & MIC & 1/2 MIC & 1.25 & ID \\
\hline AB-08 & $4 \mathrm{MIC}$ & 1/2 MIC & 4.5 & $A G$ & 1/2 MIC & MIC & 1.5 & ID & 1/2 MIC & MIC & 1.5 & ID \\
\hline AB-09 & 1/8 MIC & 1/2 MIC & 0.62 & ADD & 1/2 MIC & MIC & 1.5 & ID & 1/2 MIC & 1/2 MIC & 1.0 & ADD \\
\hline$A B-10$ & 1/2 MIC & 1/4 MIC & 0.75 & ADD & 1/2 MIC & 1/4 MIC & 0.75 & ADD & 1/2 MIC & 1/4 MIC & 0.75 & ADD \\
\hline AB-11 & MIC & 1/8 MIC & 1.125 & ID & 1/2 MIC & 1/2 MIC & 1.0 & ADD & 1/4 MIC & 1/2 MIC & 0.75 & ADD \\
\hline AB-12 & MIC & 1/4 MIC & 0.75 & ADD & 1/8 MIC & 1/2 MIC & 0.62 & ADD & 1/8 MIC & 1/2 MIC & 0.62 & ADD \\
\hline$A B-13$ & 1/2 MIC & 1/4 MIC & 0.75 & ADD & 1/8 MIC & 1/2 MIC & 0.62 & ADD & 1/2 MIC & 1/2 MIC & 1.0 & ADD \\
\hline$A B-14$ & 1/8 MIC & 1/4 MIC & 0.37 & $S$ & 1/2 MIC & 1/2 MIC & 1.0 & ADD & 1/4 MIC & 1/2 MIC & 0.62 & ADD \\
\hline AB-15 & 1/8 MIC & 1/2 MIC & 0.62 & ADD & 1/2 MIC & 1/4 MIC & 0.75 & ADD & 1/4 MIC & MIC & 1.25 & ID \\
\hline$A B-16$ & 1/16 MIC & 1/2 MIC & 0.56 & ADD & 1/2 MIC & 1/2 MIC & 1.0 & ADD & 1/4 MIC & MIC & 1.25 & ID \\
\hline AB-17 & MIC & 1/4 MIC & 1.25 & ID & 1/2 MIC & 1/4 MIC & 0.75 & ADD & 1/8 MIC & MIC & 1.125 & ID \\
\hline AB-18 & MIC & 1/4 MIC & 1.25 & ID & 1/2 MIC & 1/4 MIC & 0.75 & ADD & 1/2 MIC & 1/2 MIC & 1.0 & ADD \\
\hline$A B-19$ & 1/2 MIC & 1/2 MIC & 1.0 & ADD & 1/8 MIC & 1/4 MIC & 0.37 & $\mathrm{~S}$ & 1/2 MIC & 1/4 MIC & 0.75 & ADD \\
\hline AB- 20 & 1/4 MIC & 1/4 MIC & 0.5 & $S$ & 1/4 MIC & 1/4 MIC & 0.5 & $S$ & 1/2 MIC & 1/8 MIC & 0.62 & ADD \\
\hline
\end{tabular}

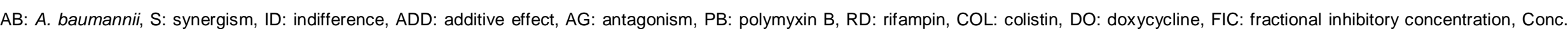
concentration.

Dijck, 2012). Antimicrobial resistance in $A$. baumannii has considerably increased in the recent past (Lockhart et al., 2007). In our study, $15 \%$ of XDR $A$. baumannii strains showed resistance to doxycycline. In a study done in 2006, doxycycline resistance was reported to be $22 \%$ for $A$. baumannii (Elmanama, 2006).

In yet another study, 8\% strains showed resistance to doxycycline (Timurkaynak et al. 2006). All strains were susceptible to polymyxin B while all of them were resistant to colistin. The susceptibility of XDR A. baumannii to polymyxin $\mathrm{B}$ is found to be $100 \%$ in other studies as well (Kuo et al., 2012; Lim et al., 2011). Colistin resistance has been reported from various regions of the world. Colistin resistance was found to be $40.6 \%$ in Spain (Arroyo et al., 2009). In Kuwait, colistin resistance was found to be $12 \%$ (Al-Sweih et al., 2011). In a study done by Chang et al. (2012) $10.4 \%$ colistin resistance was found. In another study colistin resistance was found to be $7.1 \%$ (Rodriguez et al., 2010). Although the incidence of colistin resistance is low worldwide in contrast to our findings, it has been proved through in-vitro experiment that the rate of resistance development to colisitin is rapid among Acinetobacter (Tan et al., 2007). Colistin is being used against MDR and XDR Gram negative organisms especially Pseudomonas and $A$. baumannii due to its relatively low neurotoxicity 
and nephrotoxicity as compared to polymyxin B and aminoglycosides. So far no heteroresistance has been reported in polymyxin $B$, this could be the main reason behind the $100 \%$ susceptibility to it (Mammina et al., 2012; Adams et al., 2009; Li et al., 2006) . In this study we intentionally took XDR strains of $A$. baumannii because these organisms have become a major problem and are untreatable in our setup. These isolates were resistant to colistin, however this might not represent the actual percentage of colistin resistance in our setup, as we did not take random isolates. In addition to their resistance to various antimicrobials, all XDR $A$. baumannii were resistant to tigecycline as well. Decreased susceptibility to tigecycline is linked with efflux pumps, over expressed by MDR/XDR $A$. baumannii (Ruzin et al., 2007). Tigecycline resistance among MDR A. baumannii was found to be $78 \%$ by Li et al. (2007) and $66 \%$ by Navon-Venezia et al. (2007). Al-Sweih et al. (2011) in contrast, reported $13.6 \%$ tigecycline resistance among 250 Acinetobacter isolates. The most probable reason for these contrasting results to our study could be the XDR strains in comparison with the susceptible and MRD strains which were used in the above mentioned studies. It is reported that $A$. baumannii showing resistance to multiple antimicrobial agents are notorious for reduced susceptibility to tigecycline (Ruzin et al., 2007).

Rifampin has been reported in various studies to have synergistic activity with different antibiotics against $A$. baumannii (Pachon-Ibanez et al., 2010). Thus synergistic activity of polymyxin B-rifampin in combination was found to be $15 \%$ in our study, although these strains were resistant to rifampin alone. Tan et al. (2011) reported $19 \%$ synergism in polymyxin B-rifampin combination against $A$. baumannii by the checkerboard microtitration method and $56 \%$ by the time kill assay. These results are in accordance with our study when compared with the checkerboard microtitration method. Lim et al. (2011) found the highest synergistic activity in a polymyxin Brifampin combination out of all the tested combinations $(41.9 \%)$ by time kill assay. Carl et al. (2010) reported $60 \%$ bactericidal activity in polymyxin B-doripenemrifampin triple combination against MDR $A$. baumannii in a time kill assay. All MDR $A$. baumannii strains were resistant to carbapenems and rifampin when tested alone (Urban et al., 2010). Manikal et al. (2000) found a 50\% synergistic effect of polymyxin B-rifampin combination against $A$. baumannii and a $50 \%$ additive effect by the checkerboard microtitration method. Antagonism was also noted in the polymyxin B-rifampin combination and was found to be $5 \%$, however the additive effect was found to be $55 \%$. In contrast to our study, none of the above mentioned studies reported antagonism of polymyxin B with rifampin (Tan et al., 2011; Lim et al., 2011; Urban et al., 2010).

Polymyxin B-colistin combination also showed $15 \%$ synergism. To our knowledge, a polymyxin B-colistin combination has not yet been tested against XDR $A$. baumannii. The highest additive effect was noted in this combination (65\%). Although both the antibiotic agents have the same site of action, more extensive research is needed to find an effective combination showing higher rates of synergism against XDR $A$. baumannii. In the case of the polymyxin B-doxycycline combination, only additive effect/indifference $(60 / 40 \%)$ was found. Our results are in accordance with another study in which doxycycline in combination with other antibiotics against A. baumannii showed either additive effect or indifference (Timurkaynak et al., 2006). There are several reports about the synergistic activity of polymyxin $B$ when used in combination therapies with imipenem, rifampin and azithromycin (Yoon et al., 2004; Wareham and Bean, 2006). It is noted that the results of synergy tests are highly strain and method dependent and in vitro synergy may or may not translate into in-vivo benefit (Pankey and Ashcraft, 2009).

It is concluded that polymyxin B-rifampin and polymyxin $B$-colistin combinations have demonstrated synergism against XDR $A$. baumannii by the method used, that is, checkerboard microtitration. However, the gold standard method for synergy testing is time-kill assay, that is, our study limitation. More antibiotic combinations should be tested e.g., tigecycline in order to find more effective combinations against XDR $A$. baumannii.

\section{ACKNOWLEDGEMENTS}

The authors are grateful to the University of Health Sciences, Lahore, Pakistan for providing funds for this research project. They are also thankful to Dr. Col. Raja Kamran for providing isolates for this project.

\section{REFERENCES}

Adams MD, Nickel GC, Bajaksouzian S, Lavender $H$, Murthy AR, Jacobs MR, Bonomo RA (2009). Resistance to colistin in Acinetobacter baumannii associated with mutations in the PmrAB two-component system. Antimicrob. Agents Chemother. 53(9):3628 3634.

Al-Sweih NA, Al-Hubail MA, Rotimi VO (2011). Emergence of tigecycline and colistin resistance in Acinetobacter species isolated from patients in Kuwait hospitals. J. Chemother. 23(1):13-16.

Arroyo LA, Mateos I, González V, Aznar J (2009). In vitro activities of tigecycline, minocycline, and colistin-tigecycline combination against multi- and pandrug-resistant clinical isolates of Acinetobacter baumannii group. Antimicrob. Agents Chemother. 53(3):1295-1296.

Bergogne-Berezin E, Towner KJ (1996). Acinetobacter spp. as nosocomial pathogens: microbiological, clinical, and epidemiological features. Clin. Microbiol. Rev. 9(2):148-165.

Chang KC, Lin MF, Lin NT, Wu WJ, Kuo HY, Lin TY, Yang TL, Chen YC, Liou ML (2012). Clonal spread of multidrug-resistant Acinetobacter baumannii in eastern Taiwan. J. Microbiol. Immunol. Infect. 45(1):37-42.

Davis KA, Moran KA, Mcallister CK, Gray PJ (2005). Multidrug-resistant Acinetobacter extremity infections in soldiers. Emerg. Infect. Dis. 11(8):1218-1224.

Elmanama AA (2006). Antimicrobial resistance of Acinetobacter spp. isolated from pus specimens from AL-Shifa Hospital, Gaza, Palestine. J. Al-Aqsa Unv. 10(S.E.):59-68. 
Fiori A, Van Dijck P (2012). Potent synergistic effect of doxycycline with fluconazole against Candida albicans is mediated by interference with iron homeostasis. Antimicrob. Agents Chemother. 56(7):37853796.

Giamarellou H, Antoniadou A, Kanellakopoulou K (2008). Acinetobacter baumannii: a universal threat to public health? Int. J. Antimicrob. Agents. 32(2):106-119.

Gunderson BW, Ibrahim KH, Hovde LB, Fromm TL, Reed MD, Rotschafer JC (2003). Synergistic activity of colistin and ceftazidime against multiantibiotic-resistant Pseudomonas aeruginosa in an in vitro pharmacodynamic model. Antimicrob. Agents Chemother. 47(3): 905-909.

Isenberg HD (2004). Synergism Testing: Broth Microdilution Checkerboard and broth Macrodilution Methods. In: Isenberg, H. D. ed. Clinical Microbiology Procedures Handbook. Washington, USA: ASM Press.

Karaiskos I, Galani L, Baziaka F, Giamarellou H (2013). Intraventricular and intrathecal colistin as the last therapeutic resort for the treatment of multidrug-resistant and extensively drug-resistant Acinetobacter baumannii ventriculitis and meningitis: a literature review. Int. J. Antimicrob. Agents.

Kuo SC, Chang SC, Wang HY, Lai JF, Chen PC, Shiau YR, Huang IW, Lauderdale TL (2012). Emergence of extensively drug-resistant Acinetobacter baumannii complex over 10 years: nationwide data from the Taiwan Surveillance of Antimicrobial Resistance (TSAR) program. BMC Infect. Dis. 12:200.

Li J, Nation RL, Owen RJ, Wong S, Spelman D, Franklin C (2007). Antibiograms of multidrug-resistant clinical Acinetobacter baumannii: promising therapeutic options for treatment of infection with colistinresistant strains. Clin. Infect. Dis. 45(5):594-598.

Li J, Rayner CR, Nation RL, Owen RJ, Spelman D, Tan KE, Liolios L (2006). Heteroresistance to colistin in multidrug-resistant Acinetobacter baumannii. Antimicrob. Agents Chemother. 50(9):2946-2950.

Lim TP, Tan TY, Lee W, Sasikala S, Tan TT, Hsu LY, Kwa AL (2011). In-vitro activity of polymyxin B, rifampicin, tigecycline alone and in combination against carbapenem-resistant Acinetobacter baumannii in Singapore. PLoS One 6(4):e18485.

Lockhart SR, Abramson MA, Beekmann SE, Gallagher G, Riedel S, Diekema DJ, Quinn JP, Doern GV (2007). Antimicrobial resistance among Gram-negative bacilli causing infections in intensive care unit patients in the United States between 1993 and 2004. J. Clin. Microbiol. 45(10):3352-3359.

Mammina C, Bonura C, Di Bernardo F, Aleo A, Fasciana T, Sodano C, Saporito MA, Verde MS, Tetamo R, Palma DM (2012). Ongoing spread of colistin-resistant Klebsiella pneumoniae in different wards of an acute general hospital, Italy, June to December 2011. Euro Surveill. 17(33) 1-6.

Manchanda V, Sanchaita S, Singh NP (2010). Multidrug resistant Acinetobacter. J. Glob. Infect. Dis. 2(3): 291-304.

Manikal VM, Landman D, Saurina G, Oydna E, Lal H, Quale J (2000). Endemic carbapenem-resistant Acinetobacter species in Brooklyn, New York: citywide prevalence, interinstitutional spread, and relation to antibiotic usage. Clin. Infect. Dis. 31(1):101-106.

Montefour K, Frieden J, Hurst S, Helmich C, Headley D, Martin M, Boyle DA (2008). Acinetobacter baumannii: an emerging multidrugresistant pathogen in critical care. Crit. Care Nurse 28(1):15-25.

Montero A, Ariza J, Corbella X, Doménech A, Cabellos C, Ayats J, Tubau F, Borraz C, Gudiol F (2004). Antibiotic combinations for serious infections caused by carbapenem-resistant Acinetobacter baumannii in a mouse pneumonia model. J. Antimicrob. Chemother. 54(6):1085-1091.

Navon-Venezia S, Leavitt A, Carmeli Y (2007). High tigecycline resistance in multidrug-resistant Acinetobacter baumannii. $\mathrm{J}$. Antimicrob. Chemother. 59(4):772-774.

Orhan G, Bayram A, Zer Y, Balci I (2005). Synergy tests by E test and checkerboard methods of antimicrobial combinations against Brucella melitensis. J. Clin. Microbiol. 43(1):140-143.

Pachon-lbanez ME, Docobo-Perez F, Lopez-Rojas R, DominguezHerrera J, Jimenez-Mejias ME, Garcia-Curiel A, Pichardo C, Jimenez L, Pachon J (2010). Efficacy of rifampin and its combinations with imipenem, sulbactam, and colistin in experimental models of infection caused by imipenem-resistant Acinetobacter baumannii. Antimicrob. Agents Chemother. 54(3):1165-1172.

Pankey GA Ashcraft DS (2009). The detection of synergy between meropenem and polymyxin B against meropenem-resistant Acinetobacter baumannii using Etest and time-kill assay. Diagn. Microbiol. Infect. Dis. 63(2):228-232.

Pour NK, Dusane DH, Dhakephalkar PK, Zamin FR, Zinjarde SS, Chopade BA (2011). Biofilm formation by Acinetobacter baumannil strains isolated from urinary tract infection and urinary catheters. FEMS Immunol. Med. Microbiol. 62(3):328-338.

Queenan AM, Pillar CM, Deane J, Sahm DF, Lynch AS, Flamm RK, Peterson J, Davies TA (2012). Multidrug resistance among Acinetobacter spp. in the USA and activity profile of key agents: results from CAPITAL Surveillance 2010. Diagn. Microbiol. Infect. Dis. 73(3):267-270.

Rodriguez $\mathrm{CH}$, De Ambrosio A, Bajuk M, Spinozzi M, Nastro M, Bombicino K, Radice M, Gutkind G, Vay C, Famiglietti A (2010). In vitro antimicrobials activity against endemic Acinetobacter baumannii multiresistant clones. J. Infect. Dev. Ctries. 4(3):164-167.

Rodríguez-Hernández MJ, Pachón J, Pichardo C, Cuberos L, IbáñezMartínez J, García-Curiel A, Caballero FJ, Moreno I, Jiménez-Mejías ME (2000). Imipenem, doxycycline and amikacin in monotherapy and in combination in Acinetobacter baumannii experimental pneumonia. J. Antimicrob. Chemother. 45(4):493-501.

Ruzin A, Keeney D, Bradford PA (2007). AdeABC multidrug efflux pump is associated with decreased susceptibility to tigecycline in Acinetobacter calcoaceticus-Acinetobacter baumannii complex. J. Antimicrob. Chemother. 59(5): 1001-1004.

Talbot GH, Bradley J, Edwards JE Jr, Gilbert D, Scheld M, Bartlett JG (2006). Bad bugs need drugs: an update on the development pipeline from the Antimicrobial Availability Task Force of the Infectious Diseases Society of America. Clin. Infect. Dis. 42(5):657-668.

Tan CH, Li J, Nation RL (2007). Activity of colistin against heteroresistant Acinetobacter baumannii and emergence of resistance in an in vitro pharmacokinetic/pharmacodynamic model. Antimicrob. Agents Chemother 51(9):3413-3415.

Tan TY, Lim TP, Lee WH, Sasikala S, Hsu LY, Kwa AL (2011). In vitro antibiotic synergy in extensively drug-resistant Acinetobacter baumannii: the effect of testing by time-kill, checkerboard, and Etest methods. Antimicrob. Agents Chemother 55(1):436-438.

Timurkaynak F, Can F, Azap OK, Demirbilek M, Arslan H, Karaman SO (2006). In vitro activities of non-traditional antimicrobials alone or in combination against multidrug-resistant strains of Pseudomonas aeruginosa and Acinetobacter baumannii isolated from intensive care units. Int. J. Antimicrob. Agents. 27(3):224-228.

Towner KJ (2009). Acinetobacter: an old friend, but a new enemy. J. Hosp. Infect. 73(4):355-363.

Urban C, Mariano N, Rahal JJ (2010). In vitro double and triple bactericidal activities of doripenem, polymyxin $\mathrm{B}$, and rifampin against multidrug-resistant Acinetobacter baumannii, Pseudomonas aeruginosa, Klebsiella pneumoniae, and Escherichia coli. Antimicrob. Agents Chemother. 54(6):2732-2734.

Wareham DW, Bean DC (2006). In-vitro activity of polymyxin B in combination with imipenem, rifampicin and azithromycin versus multidrug resistant strains of Acinetobacter baumannii producing OXA-23 carbapenemases. Ann. Clin. Microbiol. Antimicrob. 5:10.

Yoon J, Urban C, Terzian C, Mariano N, Rahal JJ (2004). In vitro double and triple synergistic activities of Polymyxin B, imipenem, and rifampin against multidrug-resistant Acinetobacter baumannii. Antimicrob. Agents Chemother. 48(3):753-757.

Zarrilli R, Giannouli M, Tomasone F, Triassi M, Tsakris A (2009). Carbapenem resistance in Acinetobacter baumannii: the molecular epidemic features of an emerging problem in health care facilities. J. Infect. Dev. Ctries. 3(5):335-341. 